\title{
Propriedades físico-mecânicas da madeira de canafístula aos 10 anos de idade
}

\author{
Physical and mechanical properties of 10 years old wood trees of canafístula
}

\author{
Magnos Alan Vivian ${ }^{\text {* }}$ Karina Soares Modes ${ }^{\mathrm{I}}$ Rafael Beltrame $^{\mathrm{I}}$ Joel Telles de Souza $^{\mathrm{II}}$ \\ Elio José Santini ${ }^{\mathrm{III}}$ Clóvis Roberto Haselein ${ }^{\mathrm{III}}$
}

RESUMO

A presente pesquisa objetivou caracterizar as propriedades físico-mecânicas da madeira de canafístula (Peltophorum dubium (Spreng.) Taub.) proveniente de árvores com 10 anos, por meio da determinação da massa específica básica, da retratibilidade, do módulo de elasticidade e do módulo de ruptura em flexão estática, do módulo de elasticidade em compressão perpendicular, da resistência máxima à compressão paralela à grã, da dureza de Janka, da resistência máxima ao cisalhamento e da resistência ao choque. A determinação da massa específica básica e da retratibilidade seguiu as normas COPANT 30:1-004 (1971) e 30:1-005 (1971), respectivamente, e os ensaios mecânicos seguiram as normas ASTM D 143-94 (2000) e NF B 51-009 (1942). A massa específica básica e o coeficiente de anisotropia encontrado para a espécie permitiram classificar sua madeira como leve e medianamente estável, respectivamente; já os valores médios das propriedades mecânicas encontradas definem a madeira como de resistência fraca a média. As características tecnológicas determinadas indicam que sua madeira, quando proveniente de plantios jovens, deve ser utilizada com cautela em situações que exijam elevada estabilidade dimensional e resistência mecânica, sendo, portanto, aconselhável seu corte em idades mais avançadas.

Palavras-chave: massa específica básica, retratibilidade, resistência mecânica.

\section{ABSTRACT}

This research aimed to characterize the physicmechanical properties of the canafístula wood (Peltophorum dubium (Spreng.) Taub.) from 10 years' old trees. There was determined the specific gravity, shrinkage, modulus of elasticity and modulus of rupture in static bending, modulus of elasticity in compression perpendicular to the grain, compression strength parallel to the grain, hardness, shear and impact resistance. Specific gravity and shrinkage determination were conducted according to COPANT 30:1:004 (1971) and 30:1-005 (1971), respectively, and the mechanical tests followed ASTM D 14394 (2000) and NF B51-009 (1942). The specific gravity and the ratio of tangential to radial shrinkage observed, ranked the wood as light and moderately stable, respectively, since the average values of mechanical properties classified the wood as weak to average strength. The technological characteristics observed indicate that when the wood is originated from short rotation plantation, it should be used with caution in situation which requires high dimensional stability and mechanical strength, suggesting the need of older trees.

Key words: specific gravity, shrinkage, mechanical properties.

\section{INTRODUÇÃO}

A eficiente utilização de um material, principalmente para fins estruturais, está condicionada ao conhecimento de suas propriedades físicomecânicas, tanto no que se refere aos aspectos de segurança, quanto aos de economicidade (LISBOA, 1993). A determinação das propriedades tecnológicas da madeira é importante para que se possa estimar a sua resistência às forças externas que tendem a deformá-la, bem como para definir os usos mais indicados (MELCHIORETTO \& ELEOTÉRIO, 2003).

No entanto, as características tecnológicas da madeira de uma mesma espécie variam com a

\footnotetext{
IPrograma de Pós-graduação em Engenharia Florestal (PPGEF), Centro de Ciências Rurais (CCR), Universidade Federal de Santa Maria (UFSM), 97105-900, Santa Maria, RS, Brasil. E-mail: magnosalan@yahoo.com.br. *Autor para correspondência.

${ }^{\text {IIC }}$ urso de Engenharia Florestal, CCR, UFSM, Santa Maria, RS, Brasil.

II'Departamento de Ciências Florestais (DCFL), UFSM, Santa Maria, RS, Brasil.
} 
procedência, a idade e o sítio, contribuindo para uma grande amplitude de variação nas propriedades físicomecânicas, o que demanda a realização de estudos com indivíduos em situações específicas (LADRACH, 1986). Nesse sentido, PALERMO et al. (2003) cita os efeitos da idade sobre as características de massa específica da madeira e de alta correlação com as propriedades de retratibilidade e resistência mecânica, que são parâmetros decisivos na aptidão tecnológica de uma determinada espécie.

A variabilidade da maior parte das propriedades mecânicas da madeira pode ser estimada com base na variação da densidade, que, por sua vez, está correlacionada com as dimensões das células; a densidade tende a aumentar com a idade, em função do aumento da espessura da parede celular e diminuição da largura das células (PANSHIN \& DE ZEEUW, 1980).

Verificando a influência da idade da floresta sobre a massa específica básica da madeira de Pinus taeda L. com idades de nove, 13 e 20 anos, SOUSA et al. (2007) encontraram uma elevação nos valores de massa específica básica com o aumento da idade, acompanhada sobretudo por um correspondente aumento no comprimento e na espessura dos traqueídeos.

A influência da idade sobre a retratibilidade da madeira foi abordada por SILVA et al. (2006) no estudo da madeira de Eucalyptus grandis aos 10, 14, 20 e 25 anos. Os autores observaram uma tendência de aumento desta com a idade, passando a uma redução aos 25 anos, o que se traduz num aumento da retratibilidade da madeira com o aumento da massa específica e uma redução em idades mais avançadas. O mesmo comportamento foi verificado por OLIVEIRA et al. (2006) com relação à resistência em compressão e flexão, massa específica aparente a $12 \%$ e retração volumétrica também para a madeira de Pinus taeda $L$. aos nove, 13 e 20 anos.

Nesse sentido, SERPA et al. (2003) cita que, para obtenção de madeira mais densa, estável e com maior resistência à flexão e à compressão paralela às fibras, é necessário realizar a colheita de árvores mais velhas, pois estas tendem a possuir uma porcentagem maior de madeira adulta, que apresenta, então, maior resistência, maior densidade, além de ser mais estável dimensionalmente.

No entanto, em razão do desconhecimento tecnológico por parte dos usuários da madeira, bem como da necessidade de um retorno antecipado de quem a produz, é comum o comércio de madeira de árvores jovens com uma grande percentagem de madeira juvenil, cuja participação no diâmetro do tronco depende da idade e de outros fatores que condicionam o ritmo de crescimento.

O conhecimento das espécies potencialmente produtoras de madeira de qualidade é um fator necessário para a ampliação da base florestal do Estado, que, por sua vez, é uma maneira de baixo custo, segura e de baixo impacto ambiental para gerar emprego e renda (MELCHIORETTO \& ELEOTÉRIO, 2003). Nesse contexto, a espécie Peltophorum dubium, popularmente conhecida como canafístula, se enquadra na categoria de espécie madeireira promissora, de acordo com estudo realizado pela Embrapa Florestas. Alcança um Incremento Médio Anual (IMA) de 19,60 $\mathrm{m}^{3}$ $\mathrm{ha}^{-1} \mathrm{ano}^{-1}$, portanto superior à média de $14 \mathrm{~m}^{3} \mathrm{ha}^{-1} \mathrm{ano}^{-1}$, para ser considerada de crescimento rápido (REVISTA DA MADEIRA, 2007). Segundo a mesma fonte, apresenta uma produção de madeira valiosa e desempenho silvicultural aceitável, portanto recomendável para plantios de comprovação puros ou mistos.

Sua madeira serve para construção civil e naval, parquetes, carrocerias, tábuas, tacos de assoalhos, dormentes, tornos, marcenaria, etc. (PEDROSO \& MATTOS, 1987). Conforme CARVALHO (1998) é também considerada uma espécie promissora para produção de madeira no Centro-sul do Brasil.

As propriedades mecânicas da madeira de P. dubium já foram abordadas em estudo realizado por PEDROSO \& MATTOS (1987); no entanto, com madeira de árvores adultas de diferentes procedências. Nesse sentido, é importante a condução de estudos com árvores de menor rotação e com características mais próximas da realidade de sua utilização, ampliando o conhecimento tecnológico dessa espécie.

Sendo assim, o presente estudo teve como objetivos determinar e classificar as propriedades físicomecânicas da madeira de canafístula (Peltophorum dubium (Spreng.) Taub.) aos 10 anos de idade e comparar os resultados obtidos com os encontrados por outros autores, com árvores adultas da mesma espécie.

\section{MATERIAL E MÉTODOS}

O estudo foi realizado com madeira de seis árvores de Peltophorum dubium de aproximadamente 10 anos de idade, obtidas junto à FEPAGRO FLORESTAS, localizada no Município de Santa Maria Rio Grande do Sul (RS), com diâmetro e altura média de $23,0 \mathrm{~cm}$ e $13,7 \mathrm{~m}$, respectivamente. Os ensaios físicomecânicos foram conduzidos no Laboratório de Produtos Florestais da Universidade Federal de Santa Maria (UFSM).

Ciência Rural, v.40, n.5, mai, 2010. 
Para determinação da massa específica básica, foram confeccionadas cunhas a partir de discos retirados das posições 0,$10 ; 0,30 ; 1,30 ; 3,30 ; 4,30 ; 5,30$ e $6,30 \mathrm{~m}$ de altura das árvores, seguindo os procedimentos da norma COPANT 30:1:004 (COPANT, 1971). O estudo da retratibilidade foi realizado a partir de corpos de prova confeccionados de um disco excedente da posição 1,30m (DAP) dos quais foram avaliados as contrações totais nos planos radial e tangencial das peças, a contração volumétrica e o coeficiente de anisotropia, conforme a norma COPANT 30:1-005(1971).

As amostras para realização dos testes mecânicos foram obtidas de toras de $1 \mathrm{~m}$ retiradas entre as posições $0,30 \mathrm{~m}$ e $1,30 \mathrm{~m}$. As dimensões dos corpos de prova e os procedimentos de condução destes seguiram as recomendações das normas ASTM 143-94 (ASTM, 2000) e NF B 51-009 (NFA, 1942).

Após confecção dos corpos de prova, o material foi climatizado à temperatura de $20^{\circ} \mathrm{C}$ e $65 \%$ de umidade relativa até massa constante. Foram então determinados o módulo de elasticidade (MOE) e ruptura (MOR) em flexão estática, o módulo de elasticidade
(MOE) em compressão perpendicular às fibras e a tensão no limite de proporcionalidade, a resistência máxima da madeira à compressão paralela às fibras, a dureza de Janka, a resistência máxima ao cisalhamento e a resistência ao choque.

Para auxiliar na estimativa dos valores obtidos nos testes mecânicos pelas equações de regressão, foi determinada também a massa específica aparente dos corpos de prova na condição de equilíbrio higroscópico. Os dados obtidos foram interpretados com auxílio do pacote estatístico SPSS, com nível de 95\% de significância, e os valores de resistência foram classificados de acordo com CARVALHO (1996) e MAINIERI \& CHIMELO (1987).

\section{RESULTADOS E DISCUSSÃO}

A tabela 1 apresenta um resumo das propriedades físico-mecânicas médias encontradas para a madeira de canafístula aos 10 anos de idade, com os respectivos coeficientes de variação e comparação com os citados pelo Departamento de Agricultura dos Estados Unidos (USDA, 1999).

Tabela 1 - Valores médios para a massa específica básica e aparente, retratibilidade e propriedades mecânicas da madeira de Peltophorum dubium com respectivos coeficientes de variação encontrados e aqueles informados pela USDA (1999).

\begin{tabular}{|c|c|c|c|}
\hline Propriedade & Média & $\mathrm{CV} \%$ & CV \% (USDA, 1999)1 \\
\hline $\operatorname{MEB}\left(\mathrm{g} \mathrm{cm}^{-3}\right)$ & 0,50 & 4,12 & - \\
\hline $\operatorname{MEA}\left(\mathrm{g} \mathrm{m}^{-3}\right)$ & 0,64 & 4,48 & 10 \\
\hline \multicolumn{4}{|c|}{ Retratibilidade - contração total } \\
\hline Radial (\%) & 3,30 & 17,64 & - \\
\hline Tangencial (\%) & 6,40 & 11,31 & - \\
\hline Volumétrica (\%) & 10,08 & 6,63 & - \\
\hline Coeficiente de anisotropia & 1,94 & 24,14 & - \\
\hline \multicolumn{4}{|l|}{ Flexão estática } \\
\hline MOE $\left(\mathrm{kgf} \mathrm{cm}^{-2}\right)$ & 57.389 & 19,11 & 22 \\
\hline MOR $\left(\mathrm{kgf} \mathrm{cm}^{-2}\right)$ & 773,78 & 12,14 & 16 \\
\hline \multicolumn{4}{|l|}{ Dureza Janka } \\
\hline Paralela (kgf) & 394,85 & 8,09 & \multirow[b]{2}{*}{20} \\
\hline Perpendicular (kgf) & 408,98 & 9,71 & \\
\hline \multicolumn{4}{|l|}{ Resistência ao choque } \\
\hline $\mathrm{W}(\mathrm{kgm})$ & 3,59 & 35,21 & \multirow{2}{*}{25} \\
\hline $\mathrm{K}\left(\mathrm{kgm} \mathrm{cm}^{-2}\right)$ & 0,53 & 34,02 & \\
\hline \multicolumn{4}{|c|}{ Compressão perpendicular à grã } \\
\hline MOE $\left(\mathrm{kgf} \mathrm{cm}^{-2}\right)$ & 5.220 & 22,92 & \multirow{2}{*}{28} \\
\hline Limite proporcional (kgf) & 86,5 & 17,37 & \\
\hline \multicolumn{4}{|l|}{ Compressão paralela à grã } \\
\hline Rmáx. (kgf cm²) & 309,1 & 9,30 & 18 \\
\hline \multicolumn{4}{|l|}{ Cisalhamento } \\
\hline Rmáx. (kgf cm²) & 134,86 & 10,58 & 14 \\
\hline
\end{tabular}

MEB = Massa específica aparente básica; MEA= Massa específica aparente a 15\%; MOR = Módulo de ruptura; MOE = Módulo de elasticidade; $\mathrm{W}$ = Trabalho absorvido; $\mathrm{K}$ = Coeficiente de resiliência; Rmáx = Resistência máxima; CV\% = Coeficiente de variação; ${ }^{1}$ = Valores de coeficiente de variação baseados nos resultados de testes com 50 espécies. 
Comparando-se os coeficientes de variação obtidos para a madeira de canafístula, com os valores médios para cada propriedade citados por esse órgão, percebese que, com exceção do teste de resistência ao choque, todos os demais obtidos para a madeira de canafístula foram inferiores, o que denota a maior homogeneidade dos dados obtidos nos testes e consequentemente do material utilizado. Segundo XAVIER (2008), para a área florestal e madeireira, um coeficiente de variação de até $20 \%$ é considerado baixo.

A massa específica básica longitudinal da espécie sofreu uma redução constante da base em direção ao topo, com uma amplitude de variação de $0,55 \mathrm{~g} \mathrm{~cm}^{-3}$ a $0,10 \mathrm{~m} \mathrm{e} 0,45 \mathrm{~g} \mathrm{~cm}^{-3}$ a $6,30 \mathrm{~m}$ e uma média de $0,50 \mathrm{~g} \mathrm{~cm}^{-3}$. Com base na amplitude $\left(0,13-1,90 \mathrm{~g} \mathrm{~cm}^{-3}\right) \mathrm{de}$ variação da massa específica básica em espécies florestais, segundo o IBAMA (1991), pode-se dizer que a espécie apresenta baixa densidade, traduzindo-se em boas características de usinagem.

Tomando como base a classificação dada por CARVALHO (1996) para a densidade de folhosas, a massa específica aparente encontrada para a espécie se situa no intervalo daquelas consideradas leves. PEDROSO \& MATTOS (1987), estudando as propriedades da madeira adulta de canafístula procedente de Santa Rosa-RS, observaram uma massa específica aparente a $15 \%$ de umidade de $0,77 \mathrm{~g} \mathrm{~cm}^{-3}$, já MAINIERI \& CHIMELO (1989), avaliando o comportamento da espécie Peltophorum vogelianum de procedência do Estado de São Paulo, considerada sinônimo da espécie utilizada, encontraram um valor de $0,80 \mathrm{~g} \mathrm{~cm}^{-3}$, representando valores maiores em 20 e $25 \%$, respectivamente, aos encontrados no presente estudo e passando a espécie a ser considerada pesada nesses casos. Os autores não mencionam a idade das árvores amostradas, porém pode-se também inferir que a divergência dos valores aqui encontrados para a massa específica aparente pode ser atribuída à influência da procedência do material sobre essa variável.

Com relação à retratibilidade, tomando como base CARVALHO (1996), essa madeira foi considerada de baixa contração radial e tangencial, retrátil volumetricamente e de média estabilidade dimensional. Para essas mesmas propriedades, PEDROSO \& MATTOS (1987) encontraram valores de 2,67; 7,58; 12,65 e $2,84 \%$, para a contração radial, tangencial, volumétrica e coeficiente de anisotropia, respectivamente, mudando a classificação da madeira para média contração tangencial e altamente instável dimensionalmente, o que pode ser justificado pela maior massa específica do material estudado.
Em relação às propriedades de resistência mecânica a partir da mesma fonte, pode-se inferir que o módulo de ruptura à flexão estática apresentado pela madeira de canafístula a classifica como de baixa resistência, visto que a média foi inferior a $1100 \mathrm{kgf} \mathrm{cm}^{-2}$. O módulo de elasticidade não é comentado pelo mesmo autor; no entanto, comparando-se o valor aqui obtido com o de outras madeiras estudadas por MAINIERI \& CHIMELO (1989), pode-se classificá-la como de baixa resistência quando submetida a esse tipo de esforço. Já PEDROSO \& MATTOS (1987) encontraram um valor de $114.990 \mathrm{kgf} \mathrm{cm}^{-2}$ para essa propriedade. Essa grande disparidade entre os dados pode ser consequência da menor idade das árvores testadas, refletindo numa massa específica aparente bastante inferior, o que por certo interferiu nos resultados. Quanto ao módulo de ruptura, os mesmos autores encontraram um valor de 952kgf $\mathrm{cm}^{-2}$, já MAINIERI \& CHIMELO (1989) encontraram valor de $890 \mathrm{kgf} \mathrm{cm}^{-2}$ mais próximo ao obtido e classificou-a como de resistência média.

De acordo com a faixa de resistência à dureza proposta por CARVALHO (1996) para ambos os planos de aplicação da carga, a madeira foi classificada como de dureza média, visto que permaneceram compreendidas no intervalo de 300 a 500kgf. PEDROSO \& MATTOS (1987) para essa mesma propriedade encontraram um valor de 637kgf, portanto passando a madeira a uma dureza considerada alta.

Na avaliação da espécie quanto à resistência ao choque, o trabalho absorvido encontrado a classifica como de resistência média de acordo com MAINIERI \& CHIMELO (1989). Em relação ao coeficiente de resiliência, de acordo com a classificação de CARVALHO (1996), essa madeira é considerada resistente ao choque, visto que apresentou valor no intervalo de $0,4 \mathrm{a} 1,0 \mathrm{kgm} \mathrm{cm}^{-2}$. PEDROSO \& MATTOS

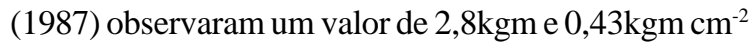
para o trabalho absorvido (W) e coeficiente de resiliência $(\mathrm{k})$, respectivamente, ou seja, todos os valores obtidos para a espécie aqui testada foram superiores.

O valor médio de MOE e o limite de proporcionalidade no teste de compressão perpendicular à grã foram inferiores aos registrados por PEDROSO \& MATTOS (1987), que encontraram valores de $123.815 \mathrm{kgf} \mathrm{cm}^{-2}$ e 266kgf, respectivamente. A resistência máxima à compressão paralela às fibras suportada pela espécie é considerada fraca de acordo com CARVALHO (1996), sendo menor que $450 \mathrm{kgf} \mathrm{cm}^{-2}$. MAINIERI \& CHIMELO (1989) e PEDROSO \& MATTOS (1987) encontraram um valor de 508 e 515kgf $\mathrm{cm}^{-2}$, respectivamente, para essa mesma propriedade, passando a ser de média resistência à ruptura. 
O valor médio de resistência máxima ao cisalhamento suportada pela espécie se encontra no intervalo de 100 a $160 \mathrm{kgf} \mathrm{cm}^{-2}$, o que a classifica como forte, superior ao obtido por PEDROSO \& MATTOS

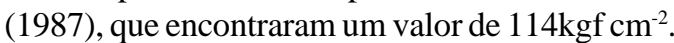

A tabela 2 traz os modelos estatísticos para estimativa das propriedades mecânicas da madeira de P. dubium em função da massa específica dos corpos de prova. Foi testada a relação da massa específica aparente com as propriedades mecânicas da madeira por meio de diversos modelos de regressão, tendo sido obtidos sempre os melhores ajustes pelos modelos lineares (maior $\mathrm{R}^{2}$ corrigido e F calculado, e menor erro padrão da estimativa). Da mesma forma, DIAS \& LAHR (2004) citam vários autores que também encontraram, no modelo linear, os melhores ajustes na estimativa das propriedades mecânicas em função da massa específica aparente.

Com relação à análise das equações obtidas para cada propriedade em função da massa específica, os melhores ajustes dos modelos estatísticos foram observados na estimativa da dureza Janka da espécie, principalmente no plano perpendicular, seguida da resistência máxima oferecida ao cisalhamento da madeira e do módulo de elasticidade à flexão estática, o que pode ter contribuído, principalmente nesta última, para os baixos valores encontrados para essa espécie em comparação com outros trabalhos, visto que a massa específica aparente ter sido bem menor, principalmente por se tratar de uma madeira de menor idade, resultado da maior proporção de lenho juvenil.
A maior relação da densidade aparente com a dureza de Janka perpendicular, em comparação com a dureza no plano paralelo, segundo XAVIER (2008), deve-se ao fato de a densidade estar ligada principalmente à espessura da parede celular e, como há uma maior proporção de parede celular por unidade de distância no sentido perpendicular às fibras, a resistência mecânica nesse sentido sofre, por consequência, maior influência dessa variável.

A não influência significativa da massa específica na obtenção das variáveis obtidas na compressão perpendicular à grã foi também encontrada por PIGOZZO (1982), estudando a madeira de peroba rosa.

Empregando o ensaio de cisalhamento às madeiras de pinho do Paraná, peroba rosa e jatobá, MENDES (1984) encontrou um bom ajuste do modelo linear relacionando a propriedade de cisalhamento e massa específica, o que também denota a alta relação entre as variáveis, como a aqui encontrada.

\section{CONCLUSÃO}

A madeira de canafístula aos 10 anos de idade é classificada como leve e medianamente estável, de acordo com sua massa específica e retratibilidade, respectivamente. As propriedades mecânicas classificam essa espécie aos 10 anos como de resistência média a fraca.

Tabela 2 - Equações ajustadas para determinação das propriedades mecânicas em função da massa específica aparente a 15\% (MEA).

\begin{tabular}{|c|c|c|c|c|}
\hline Propriedade & Equação ajustada & $\mathrm{R}^{2}$ & Syx & $\mathrm{F}$ \\
\hline \multicolumn{5}{|l|}{ Flexão estática } \\
\hline MOE $\left(\mathrm{kgf} \mathrm{cm}^{-2}\right)$ & $\mathrm{MOE}=-85.671+206.005 . \mathrm{MEA}$ & 0,4461 & $8.290,157$ & $15,994 *$ \\
\hline $\operatorname{MOR}\left(\mathrm{kgf} \mathrm{cm} \mathrm{cm}^{-2}\right)$ & MOR $=-130,89+1.302,5 \cdot \mathrm{MEA}$ & 0,2430 & 82,894 & $6,696^{*}$ \\
\hline \multicolumn{5}{|l|}{ Dureza Janka } \\
\hline Paralela (kgf cm²) & DJpar $=-104,49+792,39 \cdot \mathrm{MEA}$ & 0,7412 & 16,072 & $68,817^{*}$ \\
\hline Perpendicular $\left(\mathrm{kgf} \mathrm{cm}^{-2}\right)$ & DJperp $=-242,71+1.034,1 . \mathrm{MEA}$ & 0,8167 & 17,726 & $93,409 *$ \\
\hline \multicolumn{5}{|l|}{ Resistência ao choque } \\
\hline W (kgm) & $\mathrm{W}=-8,5334+18,958 . \mathrm{MEA}$ & 0,2763 & 1,0781 & $8,6651^{*}$ \\
\hline $\mathrm{K}\left(\mathrm{kgm} \mathrm{cm}^{-2}\right)$ & $\mathrm{K}=-0,9344+2,2376 \cdot \mathrm{MEA}$ & 0,2792 & 0,1393 & $9,5104 *$ \\
\hline \multicolumn{5}{|c|}{ Compressão perpendicular à grã } \\
\hline MOE (kgf cm²) & $\mathrm{MOE}=1759,5+5674,7 \cdot \mathrm{MEA}$ & 0,034 & $1.201,785$ & $0,785^{\mathrm{NS}}$ \\
\hline Limite proporcional (kgf) & $\mathrm{LP}=27,115+97,387 . \mathrm{MEA}$ & 0,063 & 14,870 & $1,490^{\mathrm{NS}}$ \\
\hline \multicolumn{5}{|l|}{ Compressão paralela à grã } \\
\hline Rmáx. (kgf cm²) & Rmáx = 3,2787+ 486,68.MEA & 0,4457 & 18,09 & $15,17 *$ \\
\hline \multicolumn{5}{|l|}{ Cisalhamento } \\
\hline Rmáx. (kgf cm-2) & Rmáx = -35,731 + 266,45.MEA & 0,455 & 10,633 & $21,049 *$ \\
\hline
\end{tabular}

MOE = Módulo de elasticidade; MOR = Módulo de ruptura; $\mathrm{W}=$ Trabalho absorvido; $\mathrm{K}$ = Coeficiente de resiliência; CD = Cota dinâmica; Rmáx = Resistência máxima; $\mathrm{R}^{2}=$ Coeficiente de determinação; Syx = Erro padrão da estimativa; $\mathrm{F}=$ Valor de $\mathrm{F}$ calculado; * = Significativo a $5 \%$ de significância; ${ }^{\text {NS }}=$ Não significativo. 


\section{REFERÊNCIAS}

AMERICAN SOCIETY for testing and materials. Standard methods of testing small clear specimens of timber: ASTM D 143-94. Philadelphia, 2000. 31p.

CARVALHO, A. “Madeiras portuguesas - Estrutura anatômica, propriedades, utilizações”. V.I. Instituto Florestal, 1996. 340p. Disponível em: <http://www.estv.ipv.pt/ PaginasPessoais/jqomarcelo/Tim3/tim3_TP1_Na2.pdf $>$. Acesso em: 11 out. 2009.

CARVALHO, P.E.R. Espécies nativas para fins produtivos. In: Espécies não tradicionais para plantios com finalidades produtivas e ambientais. Colombo: EMBRAPA CNPF, 1998. p.103-125.

COMISSION PANAMERICANA de normas tecnicas. Maderas: método de determinación del peso especifico aparente: COPANT 30:1-004. Caracas, 1971. 7p.

COMISSION PANAMERICANA de normas tecnicas. Maderas: método de determinación de la contracción: COPANT 30:1-005. Caracas, 1971. 5p.

DIAS, F.M., LAHR, F.A.R. Estimativa de propiedades de resistência e rigidez da madeira através da densidade aparente. Scientia Forestalis, v.1, n.65, p.102-113, 2004.

EMBRAPA FLORESTA. Espécies arbóreas brasileiras. Disponível em: <http://www.cnpf.embrapa.br/pesquisa/efb/ index_especies.htm>. Acesso em: 18 jul. 2009.

FOREST PRODUCTS LABORATORY. Wood as an engineering material. Wood handbook Gen. Tech. Rep. FPL-GTR-113. Madison, WI: U.S. Department of Agriculture, Forest Service, Forest Products Laboratory, 1999. 463p.

INSTITUTO BRASILEIRO DO MEIO AMBIENTE E DOS RECURSOS NATURAIS RENOVÁVEIS. Normas de procedimentos em estados de anatomia de madeira: $\mathrm{I}$. Angiospermae, II. Gimnospermae. Brasília: Laboratório de produtos florestais, 1991. 19p. (Série Técnica, 15).

LADRACH, W.E. Control of wood properties in plantations. In: IUFRO WORLD CONGRESS 18., 1986, Ljubljana. Proceedings... Ljubljana, Yugoslavia: IUFRO Organizing Committee, 1986. p.369-379.

L'A Association Francaise de Normalization-NFA. Norme francaise, bois essai de choc ou flexion dynamique. NF B51-009. Paris, 1942. 3p.

MAINIERI, C.; CHIMELO, J.P. Fichas de características das madeiras brasileiras. São Paulo: IPT, 1989. 418p.

MELCHIORETTO, D.; ELEOTÉRIO, J.R. Caracterização, classificação e comparação da madeira de Pinus patula, $P$. elliottii e $\boldsymbol{P}$. taeda através de suas propriedades físicas e mecânicas. In: CONGRESSO REGIONAL DE INICIAÇÃO CIENTÍFICA E TECNOLÓGICA, 18., 2003, Blumenau. Anais... Blumenau: FURB, 2003. 5p.
MENDES, A.P. Resistência da madeira ao cisalhamento. 1984. 157f. Dissertação (Mestrado em Engenharia de Estruturas) - Escola de Engenharia de São Carlos, Universidade de São Paulo, SP.

OLIVEIRA, F.L. et al. Propriedades da madeira de Pinus taeda L. em função da idade e da posição radial na tora. Revista Instituto Florestal, v.18, n. único, p.59-70, 2006. Disponível em: <http://www.iflorestal.sp.gov.br/publicacoes/Revista_if/ rev18unicopdf/trab .\%20pinus\%20taeda.pdf $>$. Acesso em: 10 out. 2009.

PALERMO, G.P.M. et al. Análise da densidade da madeira de Pinus elliottii Engelm por meio de radiação gama de acordo com as direções estruturais (longitudinal e radial) e a idade de crescimento. Floresta e Ambiente, v.10, p.47-57, 2003. Disponível em: <http://www.if.ufrrj.br/revista/pdf/ Vol10\%20no2\%2047A57.pdf>. Acesso em: 10 out. 2009.

PANSHIN, A.J.; DE ZEEUW, C. Textbook of wood technology, structure, identification, properties, and uses of the commercial woods of the United States and Canada. 4.ed. New York, McGraw-Hill, 1980. 722p.

PEDROSO, O.; MATTOS, J.R. Estudo sobre madeiras do Rio Grande do Sul. Porto Alegre: Instituto de Pesquisas de Recursos Naturais Ataliba Paz, 1987. 181p. (Publicação IPRNR, n.20).

PIGOZZO, J.C. Influência da umidade e da densidade na resistência a compressão da madeira. 1982. $141 \mathrm{f}$. Dissertação (Mestrado em Engenharia de Estruturas) - Escola de Engenharia de São Carlos, Universidade de São Paulo, SP.

SILVA, J.C. et al. Variação da retratibilidade da madeira de Eucalyptus grandis Hill ex. Maiden, em função da idade e da posição radial no tronco. Revista Árvore, v.30, n.5, p. 803810, 2006.

REVISTA DA MADEIRA. Espécies tropicais promissoras. Revista da Madeira, ano 18, n.108, p.98-106, 2007.

SERPA, P.N. et al. Avaliação de algumas propriedades da madeira de Eucalyptus grandis, Eucalyptus saligna e Pinus elliottii. Revista Árvore, v.27, n.5, p.723-33, 2003.

SOUSA, R. et al. Efeito da idade e da posição radial na densidade básica e dimensões dos traqueídeos da madeira de Pinus taeda L. Revista Instituto Florestal, v.19, n.2, p.119-127, 2007. Disponível em:<http://www.iflorestal.sp.gov.br/publicacoes/ Revista if/rev19-2pdf/Pinus\%20taeda\%20119-127.pdf>. Acesso em: 11 out. 2009.

LISBOA, C.D.J. Estudo das tensões de crescimento em toras de Eucalyptus grandis Hill Ex Maiden. 1993. $275 \mathrm{f}$. Tese (Doutorado em Ciências Florestais) - Universidade Federal do Paraná, UFPR, Curitiba, Pr.

XAVIER, R.B.L. Avaliação da dureza Janka, densidade e estabilidade de quatro espécies de Eucalyptus implantadas no estado do Rio de janeiro. 2008. 21f. Monografia, (Curso de Graduação em Engenharia Florestal) - Instituto de Florestas da Universidade Federal Rural do Rio de Janeiro, RJ. 